\title{
MODELING AND SIMULATION OF A TRAFFIC FLOW MODEL CONSIDERING THE INFLUENCE OF TRAFFIC INTERRUPTION
}

\author{
Shu-Hong Yang, Chui-Gui Li ${ }^{*}$, Dong-Xue Xia, Meng Wang, Xiao-Rong Wang \\ Department of Computer Engineering, Guangxi University of Technology, 545006, \\ Liuzhou, Guangxi, China \\ netysh2005@sina.com
}

\begin{abstract}
In this paper, we present a new lattice model of traffic flow by considering the effects of traffic interruption probability. The stability condition of the proposed model is obtained by employing the linear stability theory. The results show that the stability of traffic flow is improved by considering the influence of traffic interruption. Applying the method of nonlinear analysis, the modified Korteweg-de Vries (mKdV) equation is derived to describe the traffic behavior near the critical point. The kinkantikink soliton and the solution of the $\mathrm{mKdV}$ equation are obtained to describe the traffic jams. The good agreement between theoretical analysis and numerical simulation indicates that our new consideration can stabilize the traffic flow effectively.
\end{abstract}

Key Words- Modeling and simulation, traffic interruption probability, nonlinear analysis, stability

\section{INTRODUCTION}

In the last decades, the research of traffic flow has attracted much attention of physicists and engineers. A number of traffic models [1-16], including microscopic carfollowing models, cellular automaton (CA) models, macroscopic continuum models, and macroscopic gas-kinetic-based models, have been proposed to describe the physical mechanisms of traffic phenomena. Excellent reviews on this topic can be found in [1]. As an effective model to describe the traffic flow from macroscopic view, lattice model was first presented by Nagatani [13] in 1998. After that, many improvement have been put forward based on Nagatani's original model. In 2004, the next-nearest-neighbor interaction of traffic flow was introduced into lattice model by Xue [14]. In 2008, Li et al. [16] presented a new lattice model considering the relative current information of traffic flow. However, the lattice models mentioned above do not involve the effects that the probability of traffic interruption has on traffic flow, and thus they can not be used to describe the complex phenomena resulted by traffic interruptions. In fact, some traffic interruptions (e.g. accident) always occur with some probabilities and produce complex phenomena. Very recently, a few traffic flow models have investigated the influences of traffic interruption probability in the hydrodynamic models [11] and carfollowing models [12], and their results indicated that the consideration of the traffic interruption probability can improve the stability of traffic flow.

In this paper, we introduce another approach to study the effect of traffic interruption probability with the lattice model of traffic flow. In the following section, we review the performance of the previous lattice models of traffic flow, and then put 
forward the extended lattice model considering the effect of traffic interruption probability on a single-lane highway. In Section 3, we derived the linear stability condition by using the linear stability theory. In Section 4 , by means of nonlinear analysis, we deduce the $\mathrm{mKdV}$ equation near the critical point. In Section 5, we carry out numerical simulation to validate the analytical results. Finally, we draw some conclusions from the presented study in Section 6.

\section{MODELS}

In 1998, Nagatani [13] presented the first lattice model to describe the density wave in traffic flow on freeway. The governing equation is described as follows:

$$
\begin{aligned}
& \partial_{t} \rho+\rho_{0} \partial_{x}(\rho v)=0 \\
& \partial_{t} \rho v=a \rho_{0} V(\rho(x+\delta))-a \rho v
\end{aligned}
$$

where $\rho_{0}$ denotes the average density, $a$ is the sensitivity of a driver, $\rho(x+\delta)$ is the local density at position $x+\delta$ and time $t, \delta$ represents the average headway, which means $\delta=1 / \rho_{0}$, local density is expressed as $\rho(x+\delta)=1 / h(x, t)$, where $h(x, t)$ is the headway.

Then, the lattice model is modified with dimensionless space $\tilde{x}$ (let $\tilde{x}=x / \delta$, and $\tilde{x}$ is indicated as $x$ hereafter) and expressed as:

$$
\begin{aligned}
& \partial_{t} \rho_{j}+\rho_{0}\left(\rho_{j} v_{j}-\rho_{j-1} v_{j-1}\right)=0 \\
& \partial_{t}\left(\rho_{j} v_{j}\right)=a \rho_{0} V\left(\rho_{j+1}\right)-a \rho_{j} v_{j}
\end{aligned}
$$

where $j$ indicates the $j$ th site on a one-dimensional lattice, $\rho_{j}(t)$ denotes the local density on site $j$ at time $t$, and $v_{j}(t)$ represents its corresponding local velocity, and $V(\bullet)$ refers to the optimal velocity function. Equation (3) is the lattice version of a continuity equation, while Eq.(4) is the evolution equation.

After that, Xue [14] improved the evolution equation (4) by considering the next-nearest-neighbor interaction and described it as

$$
\rho_{j}(t+\tau) v_{j}(t+\tau)=\rho_{0} V\left(\rho_{j+1}(t)\right)(1-p)+\rho_{0} V\left(\rho_{j+2}(t)\right) p
$$

where $p$ is a constant ranging $0 \sim 0.5$, which means the front term plays the dominant role, $\tau=1 / a$ denotes the delay time. In order to further improve the stability of traffic flow, Ge et al. developed a cooperative driving lattice model of traffic flow with the evolution equation as follows [15],

$$
\rho_{j}(t+\tau) v_{j}(t+\tau)=\rho_{0} V\left(\rho_{j+1}(t), \rho_{j+2}(t), \ldots, \rho_{j+n}(t)\right)
$$

where $n$ denotes the number of sites ahead considered.

In 2008, Li et al. [16] found that the relative current information can further enhance the stability of traffic flow, and then presented a new evolution equation, i.e. 


$$
\rho_{j}(t+\tau) v_{j}(t+\tau)=\rho_{0} V\left(\rho_{j+1}\right)+k\left(\rho_{j+1} v_{j+1}-\rho_{j} v_{j}\right)
$$

However, the models mentioned above did not consider the influence of traffic interruption probability, and thus they can not be employed directly to describe the complex traffic phenomena caused by traffic interruption. In fact, the motion of vehicle may be interrupted with some probability in real traffic. Considering this, we put forward a new lattice model with the dynamic equations as follows,

$$
\begin{gathered}
\partial_{t} \rho_{j}+\rho_{0}\left(\rho_{j} v_{j}-\rho_{j-1} v_{j-1}\right)=0 \\
\rho_{j}(t+\tau) v_{j}(t+\tau)=\rho_{0} V\left(\rho_{j+1}\right)+k_{1} p\left(-Q_{j}\right)+k_{2}(1-p) \Delta Q_{j}
\end{gathered}
$$

where $p$ denotes the probability with which the preceding sites is interrupted, $k_{1}$ and $k_{2}$ are the reactive coefficients, and $V(\bullet)$ is the optimal velocity function. $Q_{j}$ is the current of $j$ th site, $\Delta Q_{j}=Q_{j+1}-Q_{j}=\rho_{j+1} v_{j+1}-\rho_{j} v_{j}$ is the relative current on site $j$. Once the leading site is completely interrupted, its current immediately becomes zero, thus the relative current between the leading site $j+1$ and following one $j$ becomes $\left(-Q_{j}\right)$. The idea of the Eq.(9) is that traffic current $\rho_{j}(t+\tau) v_{j}(t+\tau)$ on site $j$ at time $t+\tau$ is determined by the factors including optimal current $\rho_{0} V\left(\rho_{j+1}(t)\right)$, the current $Q_{j}$, the relative current $\Delta Q_{j}$ and the probability $p$. When $k_{1}=0, k_{2}=0$ and $p=0$, Eqs.(8) and (9) of the extended model reduce into those of Nagatani's model. When $k_{1}=0$ and $p=0$, Eqs.(8) and (9) of the extended model reduce into those of Li's model. Therefore, the extended model includes the Nagatani's and Li's model as special cases.

In Eq.(9), $V(\rho)$ expresses the optimal velocity function and it decreases monotonically with upper bound. We adopt the optimal velocity function as follows $[15,17]$,

$$
V(\rho)=\tanh \left(\frac{2}{\rho_{0}}-\frac{\rho}{\rho_{0}^{2}}-\frac{1}{\rho_{c}}\right)+\tanh \left(\frac{1}{\rho_{c}}\right)
$$

where $\rho_{c}=0.25$ is the critical density. Note that Eq.(10) has a turning point at $\rho_{j}=\rho_{c}$ when $\rho_{0}=\rho_{c}$.

By eliminating speed $v$ in Eqs.(8) and (9), the density equations are obtained as follows,

$$
\begin{aligned}
& \rho_{j}(t+2 \tau)-\rho_{j}(t+\tau)+\tau \rho_{0}^{2}\left[V\left(\rho_{j+1}\right)-V\left(\rho_{j}\right)\right]+k_{1} p\left[\rho_{j}(t+\tau)-\rho_{j}(t)\right] \\
& -k_{2}(1-p)\left[\Delta \rho_{j}(t+\tau)-\Delta \rho_{j}(t)\right]=0
\end{aligned}
$$

where $\Delta \rho_{j}=\rho_{j+1}-\rho_{j}$.

\section{LINEAR STABILITY ANALYSIS}

To investigate the influence of the traffic interruption probability on the traffic flow, the linear stability analysis method can be applied for the extended model as follows. It is obvious that the steady state is the uniform traffic flow with a constant density $\rho_{0}$ and optimal velocity $V\left(\rho_{0}\right)$. So, the steady-state solution is given by, 


$$
\rho_{j}(t)=\rho_{0}, v_{j}(t)=V\left(\rho_{0}, \rho_{0}, \ldots, \rho_{0}\right)
$$

Suppose $y_{j}(t)$ be a small deviation from the steady state flow,

$$
\rho_{j}(t)=\rho_{0}+y_{j}(t)
$$

Substituting Eq.(13) into Eq.(11) and linearizing them obtained

$$
\begin{aligned}
& y_{j}(t+2 \tau)-y_{j}(t+\tau)+\tau \rho_{0}^{2} V^{\prime}\left(\rho_{0}\right)\left[y_{j+1}(t)-y_{j}(t)\right] \\
& +k_{1} p\left[y_{j}(t+\tau)-y_{j}(t)\right]-k_{2}(1-p)\left[\Delta y_{j}(t+\tau)-\Delta y_{j}(t)\right]=0
\end{aligned}
$$

where $V^{\prime}\left(\rho_{0}\right)=\left.\left[d V\left(\rho_{j}\right) / d \rho_{j}\right]\right|_{\rho_{j}=\rho_{0}}$.

By expanding $y_{j}(t)=\exp (i k j+z t)$, we have the following equation of $z$ :

$$
e^{2 \tau z}-e^{\tau z}+\tau \rho_{0}^{2} V^{\prime}\left(\rho_{0}\right)\left(e^{i k}-1\right)+k_{1} p\left(e^{z \tau}-1\right)-k_{2}(1-p)\left(e^{z \tau}-1\right)\left(e^{i k}-1\right)=0
$$

Inserting $z=z_{1} i k+z_{2}(i k)^{2}+\cdots$ into equation (15) give the first and second order terms of coefficients in expression of $z_{1}$ and $z_{2}$ respectively as follows,

$$
\begin{aligned}
& z_{1}=-\frac{\rho_{0}^{2} V^{\prime}\left(\rho_{0}\right)}{1+k_{1} p} \\
& z_{2}=-\frac{\tau}{2} \frac{\left(3+k_{1} p\right)\left(\rho_{0}^{2} V^{\prime}\right)^{2}}{\left(1+k_{1} p\right)^{3}}-\frac{1}{2} \frac{\rho_{0}^{2} V_{0}^{\prime}}{\left(1+k_{1} p\right)}-k_{2} \frac{(1-p) \rho_{0}^{2} V^{\prime}}{\left(1+k_{1} p\right)^{2}}
\end{aligned}
$$

If $z_{2}$ is a negative value, the uniformly steady-state flow becomes unstable for long-wavelength modes, while the uniform flow is stable when $z_{2}$ is a positive value, thus the neutral stable criteria for this steady state is given by

$$
\tau=-\frac{\left(1+k_{1} p\right)^{2}+2 k_{2}(1-p)\left(1+k_{1} p\right)}{\left(3+k_{1} p\right) \rho_{0}^{2} V^{\prime}\left(\rho_{0}\right)}
$$

For small disturbance with long wavelengths, the homogeneous traffic flow is stable under condition that

$$
\tau<-\frac{\left(1+k_{1} p\right)^{2}+2 k_{2}(1-p)\left(1+k_{1} p\right)}{\left(3+k_{1} p\right) \rho_{0}^{2} V^{\prime}\left(\rho_{0}\right)}
$$

If the stability condition is unable to be satisfied, the uniform traffic flow will evolve into a stop and go traffic flow when affected by a small perturbation.

The neutral stability lines (solid lines) in parameter space $(\rho, a)$ are shown in Figure 1. 


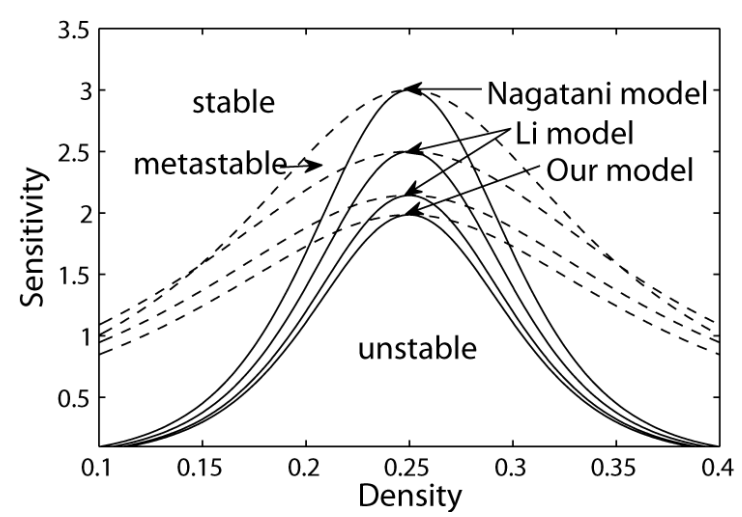

Figure 1. Phase diagram in the density-sensitivity space for Nagatani's model, Li's model with $k_{2}=0.1$ and $k_{2}=0.2$, as well as our model with $k_{l}=0.5, k_{2}=0.2, p=0.2$.

For comparison purpose, we also give out the neutral stability lines of Nagatani's and Li's model in Figure 1. On each curve, there exists the critical point $\left(\rho_{c}, a_{c}\right)$ which is the apex of the neutral stability line. Figure 1 shows that with the same value of $\rho_{c}$, the value of $a_{c}$ obtained from the proposed model is lower than those obtained from the other two models. This verifies that the stability of traffic flow can be improved by our model, and demonstrates that the factor of traffic interruption probability should not be omitted when using lattice model to describe the dynamics properties of traffic.

\section{NONLINEAR ANALYSIS AND MKDV EQUATION}

When traffic flow is affected by disturbances for various reasons, car density will fluctuate and form density waves, which is one of the causes of traffic jam. In order to further investigate the effect of traffic interruption probability on the propagation behavior of traffic jam, the reductive perturbation method is applied to the extended model described by Eq.(11). Thus, we introduce slow scales variables for space variable $j$ and time variable $t$, and define slow variable $X$ and $T$ as follows:

$$
X=\varepsilon(j+b t) \text { and } T=\varepsilon^{3} t, \quad 0<\varepsilon \leq 1
$$

where $b$ is a constant to be determined. Letting

$$
\rho_{j}(t)=\rho_{c}+\varepsilon R(X, T)
$$

where the $R(X, T)$ is a function to be determined.

Substituting Eqs.(20) and (21) into Eq.(11) and making the Taylor expansion to the fifth order of $\varepsilon$ lead to the following expression:

$$
\begin{aligned}
& \varepsilon^{2}\left[A b+\rho_{0}^{2} V^{\prime}\right] \partial_{X} R+\varepsilon^{3} A_{3} \partial_{x}^{2} R+\varepsilon^{4}\left\{\left(A \partial_{T} R+A_{41} \partial_{x}^{3} R+A_{42} \frac{V^{\prime \prime}}{6} \partial_{X} R^{3}\right\}+\right. \\
& \varepsilon^{5}\left\{\left[3 b \tau_{c}+k_{1} p-B\right] \partial_{T} \partial_{X} R+\left(A_{51}+A_{52}\right) \partial_{X}^{4} R+A_{42} \partial_{x}^{2} R^{3}+C \tau_{c} \partial_{X}^{2} R\right\}=0
\end{aligned}
$$

Where 


$$
\begin{aligned}
& V^{\prime}=\left[d V\left(\rho_{j}\right) / d \rho_{j}\right] \mid \rho_{j}=\rho_{c} \text { and } V^{\prime \prime \prime}=\left[d^{3} V\left(\rho_{j}\right) / d \rho_{j}^{3}\right] \mid \rho_{j}=\rho_{c} \\
& A=\left(1+k_{1} p\right), B=k_{2}(1-p), \text { and } C=\frac{b^{2}\left(k_{1} p+3\right)}{2} \\
& A_{3}=C \tau-B b+\rho_{0}^{2} \frac{V^{\prime}}{2} \\
& A_{41}=\frac{1}{6}\left[\rho_{0}^{2} V^{\prime}+\left(3 k_{1} p+7\right) b^{3} \tau^{2}-3 B b(b \tau+2 l+1)\right] \\
& A_{42}=\rho_{0}^{2} \frac{V^{\prime \prime}}{6} \\
& \left.A_{51}=\frac{1}{24}\left[15+k_{1} p\right) b^{4} \tau^{3}+\rho_{0}^{2} V^{\prime}-4 B\left(b^{3} \tau^{2}\right)\right] \\
& A_{52}=\frac{1}{24}\left[6 b^{2} \tau(2 l+1)+4 b\left(3 l^{2}+3 l+1\right)\right]
\end{aligned}
$$

Near the critical point $\left(\rho_{c}, a_{c}\right), \tau=\left(1+\varepsilon^{2}\right) \tau_{c}$, taking $b=-\frac{\rho_{c}^{2} V^{\prime}}{A}$ and eliminating the second order and third order terms of $\varepsilon$ from Eq.(22) result in the simplified equation,

Where

$$
\varepsilon^{4}\left[\partial_{T} R-g_{1} \partial_{X}^{3} R+g_{2} \partial_{X} R^{3}\right]+\varepsilon^{5}\left[g_{3} \partial_{X}^{2} R+g_{4} \partial_{X}^{4} R+g_{5} \partial_{X}^{2} R^{3}\right]=0
$$

$$
\begin{aligned}
& g_{1}=-\frac{1}{6 A}\left[\left(3 k_{1} p+7\right) b^{3} \tau_{c}^{2}+\rho_{0}^{2} V^{\prime}-3 B b\left(b \tau_{c}+3\right)\right] \\
& g_{2}=A_{42} \\
& g_{3}=C \tau_{c} \\
& g_{4}=A_{51}^{*}+A_{52}^{*}+\frac{b^{4} \tau_{c}^{3}}{24}-\frac{\left[3 b \tau_{c}+k_{1} p-B\right]}{A} A_{41}^{*} \\
& g_{5}=\frac{A_{42}}{2}\left(1-\frac{2\left[3 b \tau_{c}+k_{1} p-B\right]}{A}\right)
\end{aligned}
$$

It is need to note that in Eq. (34), $A_{41}^{*}, A_{51}^{*}, A_{52}^{*}$ are obtained by replacing $\tau$ with $\tau_{c}$, $l$ with 1 in Eqs. (26), (28) and (29) respectively

In order to derive the regularized equation, we make the following transformations for Eq. (30),

$$
T^{\prime}=g_{1} T, \quad R=\sqrt{\frac{g_{1}}{g_{2}}} R^{\prime}
$$

We have the standard $\mathrm{mKdV}$ equation with a $O(\varepsilon)$ correction term as follows:

where

$$
\partial_{T^{\prime}} R^{\prime}-\partial_{X}^{3} R^{\prime}+\partial_{X} R^{\prime 3}+\varepsilon M\left[R^{\prime}\right]=0
$$

$$
M\left[R^{\prime}\right]=\frac{1}{g_{1}}\left[g_{3} \partial_{X}^{2} R^{\prime}+g_{4} \partial_{X}^{4} R^{\prime}+\frac{g_{1} g_{5}}{g_{2}} \partial_{X}^{2} R^{\prime 3}\right]
$$


Ignoring the $O(\varepsilon)$ term, we will obtain the $\mathrm{mKdV}$ equation with the kinkantikink soliton solution

$$
R_{0}^{\prime}\left(X, T^{\prime}\right)=\sqrt{c} \tanh \sqrt{\frac{c}{2}}\left(X-c T^{\prime}\right)
$$

With the method described in Ref.[18], we obtain the selected velocity $C$.

$$
C=\frac{5 g_{2} g_{3}}{2 g_{2} g_{4}-3 g_{1} g_{5}}
$$

Hence, we obtain the kink-antikink soliton solution as follows:

$$
\rho_{j}(t)=\rho_{c}+\sqrt{\frac{g_{1} C}{g_{2}}\left(\frac{\tau}{\tau_{c}}-1\right)} \tanh \sqrt{\frac{C}{2}\left(\frac{\tau}{\tau_{c}}-1\right)}\left[j+\left(1-C g_{1}\left(\frac{\tau}{\tau_{c}}-1\right)\right) t\right]
$$

Then, amplitude $A$ of the kink-antikink soliton is given by

$$
A=\sqrt{\frac{g_{1} c}{g_{2}}\left(\frac{\tau}{\tau_{c}}-1\right)}
$$

The kink-antikink soliton represents coexisting phases, which consist of the freely moving phase at low density and the jammed phase at high density. The densities corresponding to the freely moving phase and the jammed phase are given, respectively, by $\rho_{j}=\rho_{c}+A$ and $\rho_{j}=\rho_{c}-A$. Thus, we can depict the coexisting curve in the $(\rho, a)$ parameter space (see the dot lines in Figure 1).

In Figure 1, the dot lines represent the coexisting curves obtained from the solution of the mKdV equation. For each pair of dot and solid lines, we can see that the phase space is divided into three different regions, i.e. stable region, metastable region and unstable region. In the stable region, traffic jam will not appear. However, in the other two areas, when small disturbances are added into the uniform traffic flow, traffic flow will evolve over time to be unstable and form density waves. In Figure 2, the propagating backward kink-antikink density wave appears which is in good agreement with the analytical results.

\section{NUMERICAL SIMULATION}

To verify the theoretical analysis above, numerical simulation was carried out with periodic boundary condition. The initial conditions in the simulation are set as follows: 
$\rho_{j}(0)=\rho_{0}=0.25, \rho_{j}(1)=\rho_{j}(0)=0.25$, for $j \neq 50,51, \rho_{j}(1)=0.25-0.1$ for $j=50$, and $\rho_{j}(1)=0.25+0.1$ for $j=51$, where the total number of sites is $N=100$, and sensitivity coefficient is $a=2.0$.

In real traffic, the traffic interruption probability depends on the related traffic condition and road configuration. For simplicity, we set the traffic interruption probability to be constant $p_{0}=0.2$ in this paper. It is need to note that we just investigate the impact of traffic interruption probability on the traffic dynamics behavior and suppose that no interruption take place in the lane really when the simulation is carried out.

Figure 2 shows the simulation results after $t=10^{4}$ time step. Figure 2(a)-2(d) show the space-time evolution of the density for Nagatani's model, Li's model with $k_{2}=0.1$, Li's model with $k_{2}=0.2$ and our model, respectively.

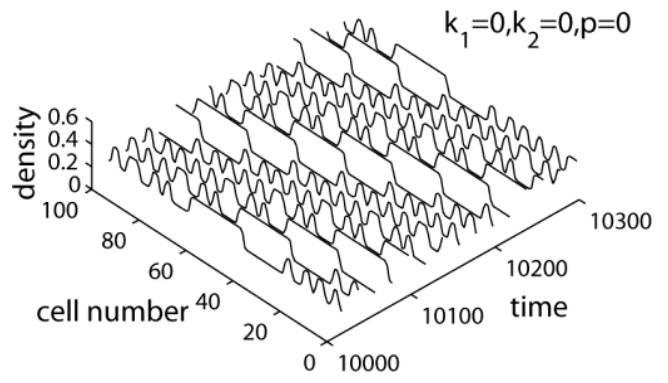

(a)

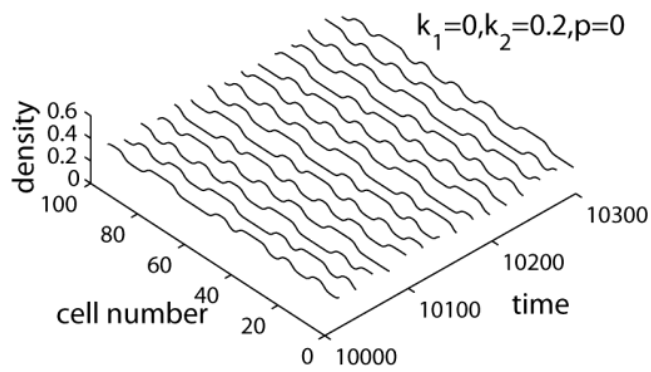

(c)

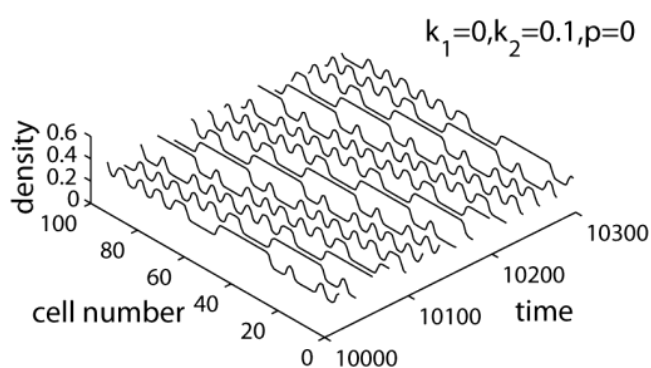

(b)

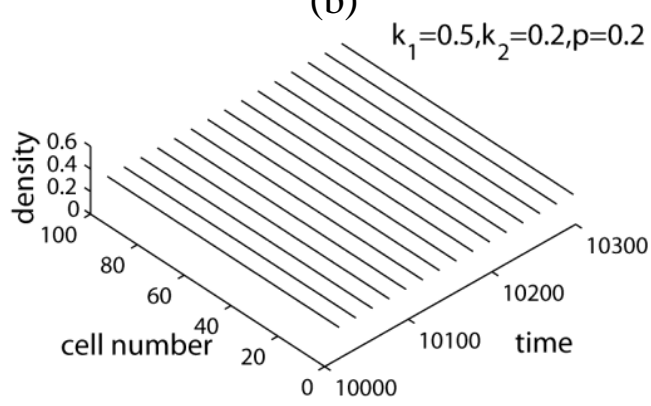

(d)

Figure 2. Space-time evolution of the density after $10^{4}$ time steps for Nagatani's model, Li's model with $k_{2}=0.1$, Li's model $k_{2}=0.2$ and our model with $k_{1}=0.5, k_{2}=0.2, p=0.2$.

In Figures 2(a)-2(c), because the stability condition are not satisfied, when small perturbation is added into the uniform traffic flow, it is amplified with time and the uniform flow changes finally to inhomogeneous traffic flow. The traffic jam in Figure 2(a) is the most serious, followed by those in Figure 2(b) and Figure 2(c) since the relative current can improve the stability of traffic flow. Nevertheless, the traffic jams still occur, which means that only considering the relative current information can not completely eliminate the fluctuation of traffic flow. In Figure 2(d), due to the probability of traffic interruption are taken into account, the perturbation finally disappears and the traffic flow recover to the uniform state.

To analyze the results of simulation quantitatively, the profile of the density wave at $t=10100$ corresponding to Figure 2. are showed in Figure 3. and its statistical results is showed in Table.1. 


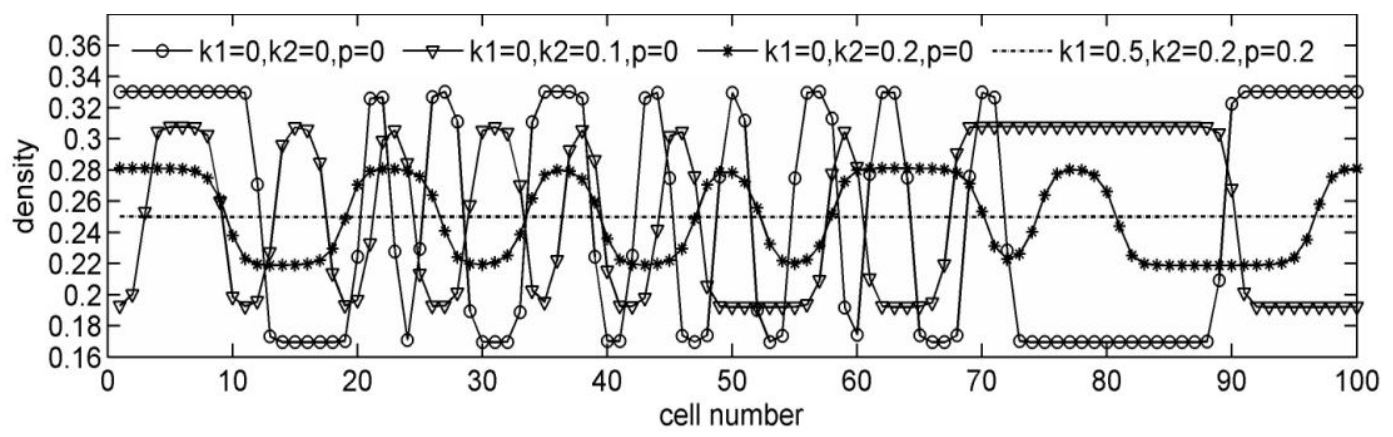

Figure 3. Profile of the density wave at $t=10100$ corresponding to Figure2 .

Table 1. Statistical results of density waves at $t=10100$ corresponding to Figure 3.

\begin{tabular}{|l|c|c|c|c|}
\hline & $\begin{array}{c}\text { Maximal } \\
\text { density }\end{array}$ & $\begin{array}{c}\text { Minimal } \\
\text { density }\end{array}$ & $\begin{array}{c}\text { Standard variance } \\
\text { of density }\end{array}$ & $\begin{array}{c}\text { Average } \\
\text { density }\end{array}$ \\
\hline (a) $\mathrm{k}_{1}=0, \mathrm{k}_{2}=0, \mathrm{p}=0$ & 0.3305 & 0.1695 & 0.0734 & 0.25 \\
\hline (b) $\mathrm{k}_{1}=0, \mathrm{k}_{2}=0.1, \mathrm{p}=0$ & 0.3079 & 0.1921 & 0.0514 & 0.25 \\
\hline (c) $\mathrm{k}_{1}=0, \mathrm{k}_{2}=0.2, \mathrm{p}=0$ & 0.2811 & 0.2188 & 0.0262 & 0.25 \\
\hline (d) $\mathrm{k}_{1}=0.5, \mathrm{k}_{2}=0.2, \mathrm{p}=0.2$ & 0.2503 & 0.2498 & 0.000137 & 0.25 \\
\hline
\end{tabular}

From Figure 3 and Table 1, we can obtain the same result with the analysis of Figure 2, that is, the fluctuation of density is decreased obviously from pattern a to patter $\mathrm{c}$, and finally disappeared in pattern $\mathrm{d}$, furthermore, this result is consistent with the analytical results in Figure 1.

Consequently, we can conclude that the probability of traffic interruption should be considered in the lattice model of traffic flow to suppress the traffic jams efficiently.

\section{SUMMARY}

In this paper, we present an extended lattice model considering the effect that the probability of traffic interruption has on traffic flow. Through the linear stability analysis, we obtain the stability criterion, and show that the new consideration can stabilize traffic flow. Moreover, the $\mathrm{mKdV}$ equation is derived to investigate the evolution behavior of traffic jams near the critical point. It should be noted that in our model and simulation, traffic interruption does not happen really but just describes the anxiety and anticipation of driver when he or she adjusts his own car according to the observed traffic flow state ahead. The good agreement between numerical simulation results and analytical ones indicated that such psychological state of drivers do influence traffic dynamics and stabilize traffic flow.

Acknowledgement- This research is supported by the High Technology Research and Development Program of China Foundation of GuangXi Zhuang autonomous region of China (Grant No. 0992006-13), and Nature Science Foundation of Guangxi University of Technology (Grant No. 0977211). 


\section{REFERENCES}

1. S. Darbha, K. R. Rajagopal, V. Tyagi, A review of mathematical models for the flow of traffic and some recent results, Nonlinear Analysis: Theory, Methods \& Applications 69, 950-970, 2008.

2. T. Chuan, S. Di-Hua, and Y. Shu-Hong, A new lattice hydrodynamic traffic flow model with a consideration of multi-anticipation effect, Chinese Physics B 20, 088902-7, 2011.

3. D. Helbing,Traffic and related self-driven many-particle systems, Reviews of Modern Physics 73, 1067-1141, 2001.

4. B. Gultekin Cetiner, M. Sari, O. Borat, A neural network based traffic-flow prediction model, Mathematical and Computational Applications 15, 269-278, 2010.

5. D.H. Sun, X.Y. Liao, G.H. Peng, Effect of looking backward on traffic flow in an extended multiple car-following model, Physica A 390, 631-635, 2011.

6. T.Q. Tang, H.J. Huang, Y. Zhang, X.Y. Xu, Stability Analysis for Traffic Flow with Perturbations, International Journal of Modern Physics C 19, 1367-1375, 2008.

7. D. Helbing, M.Treiber, A. Kesting, M. Schonhof, Theoretical vs. empirical classification and prediction of congested traffic states, European Physical Journal B 69, 583, 2009.

8. G.H. Peng, D.H. Sun, A dynamical model of car-following with the consideration of the multiple information of preceding cars, Physics Letters A 374, 1694-1698, 2010.

9. T.Q. Tang, H.J. Huang, H.Y. Shang, A new macro model for traffic flow with the consideration of the driver's forecast effect, Physics Letters A 374, 1668-1672, 2010.

10. Jamison, S., McCartney, M.: A velocity matching car-following model on a closed ring in which overtaking is allowed, Nonlinear Dynamics 58, 141-151, 2009.

11. T. Q. Tang, H. J. Huang, C. Q. Mei, S. G. Zhao A dynamic model for traffic network flow, Physica A 387, 2603-2610, 2008.

12. T. Q. Tang, H. J. Huang, S. C. Wong, R. Jiang, A new car-following model with consideration of the traffic interruption probability, Chinese Physics B 18, 975, 2009.

13. T. Nagatani., Modified KdV equation for jamming transition in the continuum models of traffic, Physica A 261,599-607, 1998.

14. Y. Xue, lattice models of the optimal traffic current, Acta Physica Sinica 53, 25-29, 2004.

15. H.X. Ge, S.Q. Dai, Y. Xue, L.Y. Dong, Stabilization analysis and modified Korteweg-de Vries equation in a cooperative driving system, Physical Review E 71, 066119-25, 2005.

16. Z.P. Li, X.L. Li, F.Q. Liu, Stabilization analysis and modified kdv equation of lattice models with consideration of relative current, International Journal of Modern Physics C 19, 1163-1173, 2008.

17. H.X. Ge, R.J. Cheng, L. Lei, The theoretical analysis of the lattice hydrodynamic models for traffic flow theory, Physica A 389, 2825-2834, 2010.

18. H.X. Ge, R.J. Cheng, S.Q. Dai, KdV and kink-antikink solitons in car-following models, Physica A 357, 466-476, 2005. 